\title{
Comorbilidad psiquiátrica y valores plasmáticos de 2-acilgliceroles en consumidores de alcohol en tratamiento ambulatorio. Análisis de las diferencias de género
}

\section{Psychiatric comorbidity and plasma levels of 2-acyl-glycerols in outpatient treatment alcohol users. Analysis of gender differences}

\begin{abstract}
Nuria García-Marchena*,*****; Pedro Araos******\#; Francisco Javier Pavón*******; Guillermo Ponce**; María Pedraz*,*****; Antonia Serrano*,*****; Francisco Arias**,*****; Pablo RomeroSanchiz*,*****; Juan SuÁrez******; Antoni Pastor****,*******; Rafael de la Torre***,****,******; Marta Torrens***,****,*****; Gabriel Rubio*,*****; Fernando Rodríguez de FonseCA******.

*Unidad Gestión Clínica de Salud Mental. Instituto de Investigación Biomédica de Málaga (IBIMA), Hospital Regional Universitario de Málaga. Málaga, Spain; **Instituto de Investigación I+12, Hospital 12 de Octubre, Psychiatry Service, Madrid 28041, Spain; Department of Psychiatry, Complutense University of Madrid, 28040, Spain; ***Neurosciences Program, Institut Hospital del Mar d'Investigacions Mèdiques (IMIM); ****Institut de Neuropsiquiatria i Addiccions (INAD) del Parc de Salut MAR. Barcelona, Spain; *****Red de Trastornos Adictivos, Instituto de Salud Carlos III; ******Facultat de Ciencies de la Salut i de la Vida, Universitat Pompeu Fabra (CEXS-UPF), Barcelona, Spain; *******Facultat de Medicina, Universitat Autónoma de Barcelona, Spain. (\#) Estos autores contribuyeron de la misma forma en este trabajo.
\end{abstract}

\section{Resumen}

Laadicción al alcohol se asocia con una elevada comorbilidad psiquiátrica que complica el tratamiento, siendo necesaria una fenotipación clínica objetiva de estos pacientes para optimizar la atención y mejorar el pronóstico. El presente estudio aborda este problema mediante los siguientes objetivos: a) estimar la prevalencia y tipos de comorbilidad psiquiátrica de una muestra de pacientes que buscan tratamiento por uso de alcohol, b) describir las diferencias de género en su presentación y c) analizar los valores plasmáticos de 2-acilgliceroles (incluyendo el endocannabinoide 2-araquidonilglicerol), estudiando su posible valor como biomarcador de alcoholismo y/o comorbilidad psiquiátrica. Para ello se reclutaron 162 pacientes evaluados con la entrevista semiestructurada PRISM, para evaluar la presencia de comorbilidad y su carácter primario o inducido. Los resultados obtenidos indican que la presencia de psicopatología se asoció a un mayor número de criterios de abuso y dependencia de alcohol Se encontraron diferencias de género tanto en la comorbilidad psiquiátrica, especialmente en trastornos del estado de ánimo. La prevalencia de comorbilidad psiquiátrica encontrada a lo largo de la vida fue del 68,5\%, destacando los trastornos del estado ánimo (37\%), y seguidos por el trastorno por déficit de atención (24,7\%, monitorizado específicamente por la entrevista WURS) y los trastornos de ansiedad (17,9\%). Entre los trastornos del estado de ánimo y psicóticos fueron más frecuentes los inducidos, mientras que en los trastornos de ansiedad los primarios fueron más prevalentes. Además, se encontraron concentraciones disminuidas significativamente de 2-acilgliceroles en pacientes con trastornos de ansiedad comórbidos diagnosticados en el último año.

Palabras clave: Comorbilidad psiquiátrica; Adicción; Alcohol; Ambulatorio; Género; 2-acilgliceroles.

\begin{abstract}
Alcohol addiction is associated with high psychiatric comorbidity. Objective stratification of patients is necessary to optimize care and improve prognosis. The present study is designed to gain insights into this challenge by addressing the following objectives: a) to estimate the prevalence of psychiatric comorbidities in a sample of outpatients seeking treatment for alcohol use disorder, b) to describe the existence of gender differences and c) to validate 2-acyl-glycerols as biomarkers of alcohol use disorder and/or psychiatric comorbidity. One hundred and sixty-two patients were recruited and evaluated with the semistructured interview PRISM. The presence of psychopathology was associated with a greater number of criteria for alcohol abuse and dependence according to DSM-IV-TR. We found gender differences in psychiatric comorbidity, e.g., mood disorder, as well as in comorbid substance use disorders. The prevalence of lifetime psychiatric comorbidity was $68.5 \%$, with mood disorders the most frequent $(37 \%)$, followed by attention deficit disorder $(24.7 \%)$ and anxiety disorders $(17.9 \%)$. Substance-induced disorders were more frequent in mood and psychotic disorders, whereas the primary disorders were more prevalent in patients with comorbid anxiety disorders. We found that 2-acyl-glycerols were significantly decreased in comorbid anxiety disorders in alcohol dependent patients in the last year, which makes them a potential biomarker for this psychopathological condition. Keywords: Psychiatric co-morbidity; Addiction; Alcohol; Outpatient; Gender; 2-acyl-glycerols.
\end{abstract}


$\mathrm{E}$ 1 alcohol es una sustancia psicoactiva con propiedades adictivas, cuyo consumo ocasiona un gran impacto económico, social y sanitario en los individuos de una población. El uso nocivo de esta sustancia es un factor etiológico de mortalidad a nivel global y hasta el año 2012, un 5,9\% de las muertes se encontraron relacionadas con el alcohol (World Health Organization, 2014). En España, las tendencias de consumos de bebidas alcohólicas en los últimos 10 años se encuentran estabilizadas, aunque las tasas de uso son elevadas. El 4,9\% de la población de 15 a 65 años presenta un consumo problemático de alcohol, y el 4,5\% presentan consumo de riesgo [1.600.000 personas, 1.300.000 hombres y 300.000 mujeres]. La edad media de inicio de consumo de alcohol se sitúa en 16,7 años y se asocia a una mayor prevalencia de consumo de otras drogas, ya que el alcohol está presente en el $90 \%$ de los policonsumos (Encuesta EDADES, 2013).

Dado que los trastornos por uso de alcohol (TUA) son frecuentes, su asociación con otras complicaciones médicas constituye un reto para los sistemas sanitarios. De entre éstas la comorbilidad psiquiátrica en el alcoholismo, es decir, la coexistencia en una misma persona de TUA y de otro trastorno mental distinto del adictivo, constituye un problema serio de salud (Goldsmith, 1999) que demanda un abordaje diferencial. Los pacientes que presentan comorbilidad psiquiátrica conforman un grupo de riesgo desde una visión clínica y social. Frecuentan en mayor medida los servicios hospitalarios (Ruffles, 2009), tienen mayores tasas de suicidios (Fiedler et al., 2012) y tienen valores de respuestas al tratamiento peores que pacientes sólo con TUA (Karila et al., 2012). Por otra parte, desde un punto de vista social son pacientes con mayor carga de conflictos a nivel laboral, judicial y de inserción social (Karila et al., 2014).

Los pacientes con TUA tienen de 2 a 4 veces más riesgo de padecer un trastorno depresivo a lo largo de su vida que aquellas personas que no presentan dependencia al alcohol (Hasin et al., 2005, 2007; Kessler et al., 1997; Ross, 1995). El estudio norteamericano National Epidemiologic Survey on Alcohol and Related Conditions (NESARC) recoge prevalencias de $39,5 \%$ en población con TUA en comparación con un $14,8 \%$ correspondiente a población general y también una elevada comorbilidad psicopatológica de TUA con trastornos del estado de ánimo (TEA), de ansiedad (TA) y de personalidad (TP) (Grant et al., 2004). Sobre los trastornos psicóticos, la prevalencia en población general sería de un $0,4 \%$ mientras que en población con TUA aumentaría a un 4,0\% (Engelhard et al., 2015).

Estos datos americanos se corresponden también con estudios realizados en Europa. En Copenhague, el 7,6\% de la población general presentaba TUA y la mitad de ellos, con un trastorno mental comórbido: TP (24\%), TEA (16,8\%) o TUS (16,6\%) (Flensborg-Madsen et al., 2009). En el año 2010, la prevalencia de TUA en nuestro país fue de 2,3\% en hombres y de 1,3\% para ambos sexos (WHO, 2014). Los trastornos derivados del uso del alcohol como la intoxicación, dependencia o los síntomas de abstinencia, empobrecen la mayoría de las relaciones sociales (Blanco et al., 2015). Los pacientes con elevada severidad en el trastorno por dependencia de alcohol, tienen alta persistencia de tener trastornos afectivos y de ansiedad (Blanco et al., 2015). Así, en un estudio efectuado en Madrid, con pacientes hospitalizados, el 24,9\% presentaban abuso de sustancias y la mayoría de ellos $(78,1 \%)$ presentaban TUA (Rodriguez-Jimenez et al., 2008).

El perfil de paciente que busca tratamiento por TUA es distinto al paciente con TUS, ya que los síntomas de abstinencia propios del TUA influyen en la búsqueda y motivación a la hora de acceder a un programa de tratamiento (Blanco et al., 2015). Por todo esto, las personas que presentan TUA tienen una mayor prevalencia de comorbilidad con otros trastornos mentales que la población general y dicha comorbilidad conlleva un TUA más grave, más discapacidad y mayor probabilidad de que soliciten tratamiento en una centro de salud mental, lo cual refuerza la necesidad de un buena detección de TUA en los pacientes atendidos en los centros de salud mental.

Tanto la comorbilidad psiquiátrica TUS como la presencia de comorbilidad psiquiátrica no relacionada con sustancias son factores que contribuyen a la progresión del abuso de alcohol hasta llegar a la dependencia de esta sustancia. En cuanto a la comorbilidad TUA y las diferencias de género, se sabe que las mujeres comienzan a beber a edades más avanzadas que ellos y desarrollan criterios de dependencia en menos tiempo que los hombres (Keyes et al., 2010). En pacientes con TUA los hombres tienen mayores prevalencias en trastornos de personalidad, mientras que ellas tienen mayor presencia de otro tipo de trastornos psiquiátricos (Ávila Escribano et al., 2007). A la luz de las diferencias de género, consideramos necesario enfatizar la importancia de establecer programas y estrategias para controlar el consumo excesivo de alcohol y la creciente ingestión de esta sustancia en la población femenina, describiendo las similitudes y diferencias en el patrón de consumo y comorbilidad psiquiátrica que pudieran darse.

Como podemos observar, son muy pocos los estudios que traten el fenómeno de la comorbilidad psiquiátrica en población con TUA en España, por tanto este trabajo podría servir de referencia para ampliar este campo del conocimiento, en especial en el entorno ambulatorio hospitalario.

Por otro parte, uno de los problemas básicos de la práctica clínica en adicciones es la carencia de pruebas biológicas objetivas que determinen el grado de consumo, la gravedad del TUS, toxicidad y respuesta a tratamiento de pacientes con estas características. Estas pruebas objetivas, biomarcadores, serían fundamentales en el diagnóstico, estratificación, pronóstico y orientación terapéutica en trastornos adictivos. Una manera de buscar biomarcadores consiste en explorar compartimentos de señalización celular que tienen 
que ver con la modulación de la respuesta a sustancias adictivas, tal y como se ha podido comprobar en pacientes consumidores de cocaína (Araos et al., 2014; Pavón et al., 2013; Pedraz et al., 2015). En el presente estudio nos centraremos en el estudio de moléculas de señalización denominadas acilgliceroles, que son ésteres formados a partir de ácidos grasos, las moléculas más conocidas de este tipo incluyen al 2-araquidonil-glicerol (2-AG) y el 2-linolil-glicerol (2-LG). El 2-AG es el principal endocannabinoide endógeno y su actividad está ligada a la preferencia alcohólica así como al desarrollo de tolerancia, como demuestran múltiples estudios en modelos preclínicos (Basavarajappa et al., 2005; Caillés et al., 2007; Malinen et al., 2009; Serrano et al., 2012). Estos compuestos están presentes en el sistema nervioso central con niveles incluso más abundantes que otros transmisores lipídicos como las aciletanolamidas, con las que comparten su perfil cannabinoide endógeno (Piomelli, 2003).

En resumen, el presente estudio se ha diseñado para valorar la prevalencia en pacientes con TUA que han solicitado atención ambulatoria de comorbilidad psiquiátrica, describiendo las diferencias de género y determinando los valores plasmáticos de acilgliceroles y su valor como biomarcadores.

\section{Método}

\section{Diseño y población objeto del estudio}

En el año 1994 se creó, en el Hospital 12 de Octubre de Madrid, un programa específico para dar una respuesta integral al paciente con TUA en forma de unidad mixta médico-psiquiátrica (pacientes aunados por los servicios de Psiquiatría, Medicina Interna y Digestivo). En esta unidad se ha procedido al reclutamiento de pacientes para realizar un estudio observacional transversal y descriptivo con el objetivo de determinar la prevalencia de las comorbilidades psiquiátricas en sujetos que acuden en busca de tratamiento y de obtener una muestra biológica de plasma para la validación de los 2-acilgliceroles como biomarcadores.

El estudio se realizó con una muestra de 262 participantes divididos en dos grupos: pacientes en abstinencia que acudieron a tratamiento ambulatorio por deshabituación de alcohol en el programa de trastornos relacionados con las conductas adictivas del Hospital Universitario 12 de Octubre de Madrid por un diagnóstico de TUA, y sujetos control sin diagnóstico de abuso y/o dependencia de sustancias legales e ilegales y sin antecedentes de diagnóstico psiquiátrico concomitante (ambos según criterios diagnósticos DSM-IV-TR). Se incluyeron en el estudio de evaluación clínica, 162 pacientes que participaban en el programa anteriormente descrito, de los cuales 133 consintieron donar una muestra biológica, además de, 100 sujetos controles emparejados por edad, género e índice de masa corporal.

Para el cálculo del tamaño muestral se tomó como referencia el trabajo de Pérez-Gálvez y colaboradores (Pé-
rez-Gálvez et al., 2008), en el que la prevalencia de comorbilidad psiquiátrica en pacientes con TUA es de un $70 \%$ aproximadamente. Para conseguir una precisión del $8 \%$ en la estimación de una proporción mediante un intervalo de confianza asintótico normal al 95\% bilateral, asumiendo que la proporción es del $70 \%$, será necesario incluir para este estudio una muestra de 160 sujetos aproximadamente. Dados los objetivos del estudio, en la selección de los sujetos de la muestra se adopta una actitud pragmática. Por ello se emplean unos criterios no restrictivos con el propósito de maximizar la representatividad de la muestra seleccionada y la extrapolación de los resultados del estudio. Se aplicó un muestreo consecutivo no aleatorio, reclutando a los pacientes según acudían a la unidad ambulatoria hospitalaria y tras cumplir los criterios de selección.

Los criterios de inclusión que se tuvieron en cuenta para la participación en el estudio fueron: ser paciente del programa y estar en tratamiento, en fase de deshabituación por TUA y con una abstinencia de alcohol mínima de 30 días, y estar dispuesto a participar firmando el consentimiento informado. Los criterios de inclusión que se tuvieron en cuenta para la participación en el estudio para los sujetos controles fueron: no tener historia de abuso y/o dependencia de sustancias, comorbilidad psiquiátrica concomitante y consentimiento informado firmado.

Los criterios de exclusión que se tuvieron en cuenta para los pacientes en abstinencia y también para los sujetos controles fueron: la presencia de alteraciones cognitivas que dificultasen la aplicación de los instrumentos de evaluación diagnóstica y el rechazo de participar en el estudio por parte del paciente.

Los aspectos éticos del presente estudio fueron aprobados por el comité de ética en Investigación Clínica del Hospital Universitario 12 de Octubre de Madrid. Todos los pacientes fueron informados y se reclutaron aquellos que firmaron el consentimiento informado aprobado por dicho comité. Este estudio está enmarcado en el programa "Consecuencias médicas del alcoholismo" de la Red de Trastornos Adictivos del Instituto de Salud Carlos III.

El proceso de evaluación fue realizado por un psicólogo sanitario general con formación especializada y acreditada en evaluación psicopatológica.

\section{Procedimiento}

Se contó con la colaboración de los psiquíatras y enfermeras del programa para informar a los pacientes en consulta y en terapia de grupo acerca de la existencia del estudio previo su consentimiento, si estos cumplían los criterios de inclusión establecidos. Una vez citado el paciente y/o el sujeto control y firmado el consentimiento se realizaba la evaluación clínica en el Centro de Actividades Ambulatorias del Hospital Universitario 12 de Octubre de Madrid.

El proceso de evaluación psicopatológica se realizaba en una misma mañana y podía durar de 1 hasta 2 horas, en 
consulta individual. Las entrevistas se realizaron durante los meses de Octubre del 2013 hasta marzo del 2015, y al finalizar cada entrevista física se registraban los datos en una base de datos diseñada para este estudio.

\section{Instrumentos de Medida}

PRISM. La entrevista diagnóstica PRISM (Psychiatric Research Interview for Substance and Mental Diseases) es el principal instrumento utilizado para valorar los trastornos psiquiátricos y por sustancias. Se trata de una entrevista clínica semiestructurada diseñada para resolver los problemas de diagnóstico en personas con un consumo elevado de sustancias y/o alcohol, evaluando en un primer módulo preguntas del historial de consumo del sujeto, arrojando diagnóstico de abuso y dependencia tanto en el último año como en el pasado. Además, evalúa 20 trastornos del Eje I y los dos trastornos del Eje II más prevalentes en esta población: el trastorno límite de la personalidad y el trastorno antisocial de la personalidad. Los diagnósticos se realizan en dos marcos temporales de evaluación. Por un lado, la entrevista valora los trastornos actuales, es decir, los que están presentes durante el último año. Por el otro, evalúa los trastornos previos, es decir, los definidos con anterioridad al último año. De manera general, la prevalencia diagnóstica presentada por un sujeto "a lo largo de la vida" incluiría la totalidad de los diagnósticos observados en ambos periodos temporales, es decir, los actuales más los previos.

Una de las características más importantes de este instrumento es que permite diferenciar los trastornos inducidos por sustancias, así como los síntomas esperados del efecto de la intoxicación y la abstinencia. El criterio establecido por la PRISM para que un trastorno psiquiátrico pueda ser considerado inducido por la sustancia, es que debe ocurrir en el contexto de un consumo patológico de la sustancia, en cualquiera de estas dos situaciones: a) intoxicación crónica, es decir, un consumo de cuatro o más días a la semana durante un mes; b) atracones, durante un periodo de consumo de tres días continuados. Para diferenciar los síntomas psiquiátricos inducidos de los esperados durante la intoxicación o la abstinencia, se debe determinar si hubo un cambio brusco en el patrón de consumo (Hasin et al., 1996; Torrens et al., 2004).

Esta entrevista presenta buena fiabilidad test-retest, validez y fiabilidad inter-examinadores (coeficiente Kappa oscila entre 0,66 y 1,00) (Morguello et al., 2006).

WURS. Debido al especial interés existente entre la asociación del TUA y el trastorno por déficit de atención e hiperactividad (TDAH), se procedió a evaluar la prevalencia del TDAH utilizando un instrumento adicional, la prueba WURS (Wender-Utah Rating Scale). Esta prueba se utiliza para la evaluación retrospectiva del diagnóstico de TDAH en pacientes adultos. Se trata de un cuestionario auto-aplicado de 61 ítems, entre los que se han seleccionado 25 por su capacidad para discriminar a los pacientes adultos, con antecedentes de
TDAH en la infancia, de otras poblaciones como controles y depresivos. La WURS ha demostrado una consistencia interna y una estabilidad temporal satisfactorias en varios trabajos. Se recogen preguntas sobre estados de ánimo, problemas de relación con familiares, compañeros y figuras de autoridad, problemas médicos, escolares y académicos. El coeficiente de Cronbach para esta subescala fue de 0,94. El punto de corte de 32 optimizaba sensibilidad $(91,5 \%)$.

CIDI. Para la evaluación psiquiátrica de los sujetos control se utilizó la versión española de la Composite Internacional Diagnostic Interview (CIDI) (Robins et al., 1988). Esta entrevista estructurada tiene una sección que evalúa 22 diagnósticos de distintos trastornos en forma de cribado que evalúa entre otros trastornos de inicio en la infancia y otros trastornos (como trastornos psicóticos) (Kessler y Ustün, 2004). Además, para la evaluación de trastornos de los controles se utilizó el apartado de historial de uso de drogas de la entrevista PRISM.

\section{Procesamiento de sangre y obtención de muestras plasmáticas}

Las muestras de sangre periférica de los 233 donantes (133 pacientes en abstinencia y 100 sujetos control) se obtuvieron por la mañana tras 8-12 horas de ayuno (el mismo día de la entrevista). Se extrajeron $10 \mathrm{~mL}$ de sangre periférica utilizando tubos BD vacutainer® con K2 EDTA por el personal de enfermería colaborador en el estudio. Para obtener el plasma, las muestras se centrifugaron a temperatura ambiente a $2200 \mathrm{xG}$ durante 15 minutos. Para descartar la presencia de enfermedades infecciosas (SIDA-VIH, hepatitis B y C) se realizaron pruebas inmediatas para cada una de las muestras. Cada una de las muestras de plasma se registró y caracterizó de forma individual, descartando aquellas que mostraran algún tipo de infección tras el uso de los protocolos de seguridad. Finalmente, todas las muestras se almacenaron a $-80^{\circ} \mathrm{C}$ para su posterior análisis. El tiempo entre extracción y congelación del plasma nunca superó los 30 minutos.

\section{Análisis de las concentraciones plasmáticas de 2-acilgliceroles}

Las muestras de plasma fueron procesadas según técnicas descritas tras una extracción orgánica de los lípidos del plasma (Pavón et al., 2013). La separación cromatográfica se realizó con una columna Zorbax StableBond 80 Å C8 (2,1 x $100 \mathrm{~mm}, 1,8$ micras de tamaño de partícula) mantenida a $40^{\circ} \mathrm{C}$ con un caudal de la fase móvil de $0,4 \mathrm{ml} / \mathrm{min}$. La composición de la fase móvil fue: A) 0,1\% (v/v) de ácido fórmico en agua. B) 0,1\% (v/v) de ácido fórmico en acetonitrilo. Las condiciones iniciales fueron de un $40 \%$ de B. El gradiente se incrementó linealmente hasta $100 \%$ de B durante 4 minutos, mantenido a $100 \%$ de B durante 4 minutos, y regresaron a las condiciones iniciales de unos 5,5 minutos más con un tiempo total de 13,5 minutos. El espec- 
trómetro de masas cuadrupolar en tándem operado en el modo de electropulverización positiva. Una temperatura de gas de desolvatación de $350^{\circ} \mathrm{C}$ y una velocidad de flujo de gas de $10 \mathrm{~L}$ se utilizaron / min. La presión del nebulizador se fijó en 40 psi y la tensión capilar a 4.000 V. El fragmentador se fijó en $135 \mathrm{~V}$ y el tiempo de permanencia a $20 \mathrm{~ms}$ para todos los analitos. El modo de monitorización de reacciones múltiples se utilizó para el análisis con la siguiente precursor a las transiciones de iones producto: $\mathrm{m} / \mathrm{z} 379.2$ / 287 para el 2- AG, $\mathrm{m}$ / z 384.3/287 para el 2- AG- d5 y m / z 355.2/263 para 2 -LG.

\section{Descripción del estudio}

El estudio descriptivo abordó los siguientes objetivos: La existencia de diferencias de género en las características socio-demográficas y patrones de uso de alcohol; la prevalencia de comorbilidad psiquiátrica TUA y TUS (abuso y/o dependencia) en los pacientes reclutados; la prevalencia de comorbilidad psiquiátrica no TUS y finalmente el análisis de las concentraciones plasmáticas de 2-acilgliceroles en esta población, comparándola con la población control, y determinando las asociaciones existentes con la comorbilidad psiquiátrica de los pacientes reclutados.

\section{Análisis estadístico}

Los datos se expresaron mediante números, porcentaje de sujetos $[\mathrm{n}(\%)]$, medias y desviaciones estándar (DE). Las diferencias en las variables categóricas se determinaron mediante la prueba exacta de Fisher o la prueba de Chi-cuadrado $\left(\chi^{2}\right)$ mientras que las variables continuas se evaluaron mediante diferentes enfoques estadísticos de acuerdo con el número de comparaciones y la distribución de las variables. Para las comparaciones de dos grupos, se utilizó el estadístico t de Student (t-test) para las variables continuas con distribución normal y el test de Mann-Whitney como prueba no paramétrica. Para las comparaciones de tres o más grupos, se utilizó el análisis Kruskal-Wallis y para los análisis no paramétricos se utilizó el estadístico post-test Dunn. La distribución normal de las variables fue evaluada utilizando el test de normalidad general de D Agostino y Pearson. El valor $p$ menor a 0,05 fue considerado estadísticamente significativo.

Para los análisis estadísticos se utilizaron el programa estadísticos SPSS versión 19.0 (SPSS Inc., an IBM Company) y la versión 5.04 del Graph-Pad Prism (GraphPad Software, San Diego, CA, USA).

\section{Resultados}

\section{Características socio-demográficas y diferencias de género.}

La descripción de los resultados socio-demográficos de la muestra de 162 sujetos se encuentra en la Tabla 1.

En relación a las variables socio-demográficas, en función de las diferencias de género, podemos decir que el
$75,3 \%$ de la muestra estuvo compuesto de hombres y un $24,7 \%$ de mujeres, sin diferencias en cuanto a la edad [media: 49,3 años $(\mathrm{DE}=8,1)$ ]. El índice de masa corporal (IMC) fue muy significativamente $(* \mathrm{p}<0,01)$ distinto entre géneros, siendo menor en mujeres [media: 24,1 $(\mathrm{DE}=3,9)$ ] que en hombres [media: 26,8 $(\mathrm{DE}=4,5)]$. Otras diferencias muy significativas en relación al género $\left({ }^{*} \mathrm{p}<0,01\right)$ se dieron en antecedentes judiciales y medicación psiquiátrica. Los hombres tienen más antecedentes judiciales, y ellas toman más medicación psiquiátrica que ellos. Hay diferencias significativas $(* \mathrm{p}<0,05)$ en el estado civil, donde el $41 \%$ de los hombres y el $25 \%$ de mujeres estaban casados. También hubo diferencias significativas $(* \mathrm{p}<0,05)$ en los trastornos por uso de sustancias (TUS), donde el $25 \%$ de las mujeres presentaban comorbilidad TUS frente al 44,3\% de los hombres. Y por último, hubo diferencias significativas $(* \mathrm{p}<0,05)$ en comorbilidad psiquiátrica de otros trastornos no relacionados con uso de sustancias donde el $82,5 \%$ de las mujeres presentaban comorbilidad frente al $63,9 \%$ de los hombres en esta variable.

\section{Patrones de uso de alcohol en función del género y comorbilidad psiquiátrica.}

Las variables relacionadas con los patrones de uso de alcohol y las diferencias de género y comorbilidad psiquiátrica distinta de los trastornos de sustancias se encuentran reflejadas en la Tabla 2.

Encontramos diferencias muy significativas $\left({ }^{*} \mathrm{p}<0,01\right)$ en la variable edad del primer del consumo de alcohol, donde las mujeres [media 18,2 años $(\mathrm{DE}=3,2)$ ] tardan más años en probar el alcohol que los hombres [media 15,92 años $(\mathrm{DE}=3,6)]$. También habría diferencias significativas $(* \mathrm{p}<0,05)$ en la variable edad de inicio del consumo problemático en género y comorbilidad psiquiátrica. En esta ocasión, entre el grupo de hombres con comorbilidad psiquiátrica [media 25,73 años $(\mathrm{DE}=9,8)$ ] y el mujeres con comorbilidad psiquiátrica [media 32,5 años $(\mathrm{DE}=12,9)$ ]. Estos datos nos dicen que ellas probaron el alcohol más tarde y también comenzaron el consumo problemático a edades más avanzadas.

También se ven diferencias muy significativas $\left({ }^{*} \mathrm{p}<0,001\right)$ en los años de duración del consumo problemático, y en este caso también tenemos diferencias significativas $(* \mathrm{p}<0,05)$ entre los dos grupos de comorbilidad psiquiátrica con trastornos no relacionados con sustancias. Los hombres con otra comorbilidad psiquiátrica llevan más años de enfermedad [media 15,3 años $(\mathrm{DE}=6,9)]$ con respecto a las mujeres con comorbilidad psiquiátrica [media 11,2 años $(\mathrm{DE}=6,3)$ ] antes de buscar un tratamiento para el TUA. Por último, hay diferencias muy significativas $\left({ }^{*} \mathrm{p}<0,001\right)$ en el número de criterios de adicción del episodio de máxima severidad. Concretamente, encontramos diferencias muy significativas $\left({ }^{*} \mathrm{p}<0,001\right)$, entre el grupo de hombres con comorbilidad psiquiátrica y los hombres sin comorbilidad, en cuanto al 
Tabla 1. Variables socio-demográficas en función del sexo de una población de consumidores de alcohol en tratamiento.

\begin{tabular}{|c|c|c|c|c|c|}
\hline Variables & & Total & Hombres & Mujeres & Valor $p$ \\
\hline $\begin{array}{l}\text { Número de pacientes } \\
{[N(\%)]}\end{array}$ & & $162(100)$ & $122(75,3)$ & $40(24,7)$ & 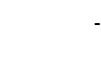 \\
\hline $\begin{array}{l}\text { Edad } \\
\text { [media (DE)] }\end{array}$ & & $49,3(8,1)$ & $49,1(8,5)$ & $49,9(6,9)$ & $0,575^{1}$ \\
\hline $\begin{array}{l}\text { Índice de masa corporal } \\
\text { [media (DE)] }\end{array}$ & & $26,2(4,5)$ & $26,8(4,5)$ & $24,1(3,9)$ & $<0,001^{1}$ \\
\hline $\begin{array}{l}\text { Estado civil } \\
{[\mathrm{N}(\%)]}\end{array}$ & $\begin{array}{l}\text { Soltero } \\
\text { Casado } \\
\text { Separado/Divorciado } \\
\text { Viudo }\end{array}$ & $\begin{array}{r}47(29,0) \\
60(37,0) \\
51(31,5) \\
4(2,5)\end{array}$ & $\begin{array}{r}34(27,9) \\
50(41,0) \\
37(30,3) \\
1(0,8)\end{array}$ & $\begin{array}{r}13(32,5) \\
10(25,0) \\
14(35,0) \\
3(7,5)\end{array}$ & $<0,05^{2}$ \\
\hline $\begin{array}{l}\text { Nivel educativo } \\
{[N(\%)]}\end{array}$ & $\begin{array}{l}\text { Sin estudios } \\
\text { Primaria } \\
\text { Secundaria } \\
\text { Universidad }\end{array}$ & $\begin{array}{r}4(2,5) \\
53(32,7) \\
83(51,2) \\
22(13,6)\end{array}$ & $\begin{array}{r}3(2,5) \\
45(36,9) \\
60(49,2) \\
14(11,5)\end{array}$ & $\begin{array}{r}1(2,5) \\
8(20,0) \\
23(57,5) \\
8(20,0)\end{array}$ & $0,200^{2}$ \\
\hline $\begin{array}{l}\text { Situación laboral } \\
{[N(\%)]}\end{array}$ & $\begin{array}{l}\text { Empleado } \\
\text { Baja por enfermedad } \\
\text { Desempleado/Invalidez } \\
\text { Jubilación } \\
\text { Labores domésticas }\end{array}$ & $\begin{array}{r}46(28,4) \\
27(16,7) \\
68(42,0) \\
17(10,5) \\
\quad 4(2,5)\end{array}$ & $\begin{array}{r}36(29,5) \\
23(18,9) \\
49(40,2) \\
14(11,5) \\
-\end{array}$ & $\begin{array}{r}10(25,0) \\
4(10,0) \\
19(47,5) \\
3(7,5) \\
4(10,0)\end{array}$ & $0,466^{2}$ \\
\hline $\begin{array}{l}\text { Antecedentes judiciales } \\
{[\mathrm{N}(\%)]}\end{array}$ & $\begin{array}{l}\text { Sí } \\
\text { No }\end{array}$ & $\begin{array}{r}40(24,7) \\
122(75,3)\end{array}$ & $\begin{array}{l}37(30,3) \\
85(69,7)\end{array}$ & $\begin{array}{r}3(7,5) \\
37(92,5)\end{array}$ & $<0,01^{2}$ \\
\hline $\begin{array}{l}\text { Otro problema médico } \\
{[\mathrm{N}(\%)]}\end{array}$ & $\begin{array}{l}\text { No } \\
\text { Endocrino } \\
\text { Circulatorio } \\
\text { Digestivo } \\
\text { Sistema nervioso }\end{array}$ & $\begin{array}{r}89(54,9) \\
8(4,9) \\
23(14,2) \\
33(20,4) \\
9(5,6)\end{array}$ & $\begin{array}{r}65(53,3) \\
4(3,3) \\
20(16,4) \\
27(22,1) \\
6(4,9)\end{array}$ & $\begin{array}{r}24(60,0) \\
4(10,0) \\
3(7,5) \\
6(15,0) \\
3(7,5)\end{array}$ & $0,215^{2}$ \\
\hline $\begin{array}{l}\text { Procedencia del paciente } \\
{[N(\%)]}\end{array}$ & $\begin{array}{l}\text { Psiquiatría } \\
\text { Digestivo/Medicina interna } \\
\text { Centro de salud mental } \\
\text { Otros }\end{array}$ & $\begin{array}{r}120(74,1) \\
35(21,6) \\
5(3,1) \\
2(1,2)\end{array}$ & $\begin{array}{r}86(70,5) \\
4(3,3) \\
30(24,6) \\
2(0,16)\end{array}$ & $\begin{array}{r}34(85,0) \\
1(2,5) \\
5(12,5) \\
-\end{array}$ & $0,192^{2}$ \\
\hline $\begin{array}{l}\text { Tratamiento } \\
\text { psiquiátrico inicial } \\
{[\mathrm{N}(\%)]}\end{array}$ & $\begin{array}{l}<1 \text { año } \\
1-5 \text { años } \\
5-10 \text { años } \\
>10 \text { años }\end{array}$ & $\begin{array}{r}38(23,5) \\
46(28,4) \\
16(9,9) \\
62(38,3)\end{array}$ & $\begin{array}{l}29(23,8) \\
36(29,5) \\
15(12,3) \\
42(34,4)\end{array}$ & $\begin{array}{r}9(22,5) \\
10(25,0) \\
1(2,5) \\
20(50,0)\end{array}$ & $0,167^{2}$ \\
\hline $\begin{array}{l}\text { Medicación psiquiátrica } \\
{[N(\%)]}\end{array}$ & $\begin{array}{l}\text { Sí } \\
\text { No }\end{array}$ & $\begin{array}{r}123(75,9) \\
39(24,1)\end{array}$ & $\begin{array}{l}86(70,5) \\
36(29,5)\end{array}$ & $\begin{array}{r}37(92,5) \\
3(7,5)\end{array}$ & $<0,01^{2}$ \\
\hline $\begin{array}{l}\text { Antecedentes familiares } \\
\text { con adicciones } \\
{[\mathrm{N}(\%)]}\end{array}$ & $\begin{array}{l}\text { Sí } \\
\text { No }\end{array}$ & $\begin{array}{r}100(61,7) \\
62(38,3)\end{array}$ & $\begin{array}{l}45(36,9) \\
77(63,1)\end{array}$ & $\begin{array}{l}17(42,5) \\
23(57,5)\end{array}$ & $0,576^{2}$ \\
\hline $\begin{array}{l}\text { Fumador } \\
{[\mathrm{N}(\%)]}\end{array}$ & $\begin{array}{l}\text { Sí } \\
\text { Ex-fumador } \\
\text { No }\end{array}$ & $\begin{array}{r}111(68,5) \\
26(16,0) \\
25(15,4)\end{array}$ & $\begin{array}{l}80(65,6) \\
23(18,9) \\
19(15,6)\end{array}$ & $\begin{array}{r}31(77,5) \\
3(7,5) \\
6(15,0)\end{array}$ & $0,218^{2}$ \\
\hline $\begin{array}{l}\text { Trastornos por uso de } \\
\text { sustancias (TUS) } \\
{[\mathrm{N}(\%)]}\end{array}$ & $\begin{array}{l}\text { Solo TUA } \\
\text { TUA + otros TUS }\end{array}$ & $\begin{array}{l}98(60,4) \\
64(39,6)\end{array}$ & $\begin{array}{l}68(55,7) \\
54(44,3)\end{array}$ & $\begin{array}{l}30(75,0) \\
10(25,0)\end{array}$ & $<0,05^{2}$ \\
\hline $\begin{array}{l}\text { Otros trastornos de } \\
\text { comorbilidad psiquiátrica* } \\
{[\mathrm{N}(\%)]}\end{array}$ & $\begin{array}{l}\text { Sí } \\
\text { No }\end{array}$ & $\begin{array}{r}111(68,5) \\
51(31,5)\end{array}$ & $\begin{array}{l}78(63,9) \\
44(36,1)\end{array}$ & $\begin{array}{r}33(82,5) \\
7(17,5)\end{array}$ & $<0,05^{2}$ \\
\hline
\end{tabular}

Nota. Abreviaturas: $\mathrm{N}=$ =número de sujetos; $\mathrm{DE}=$ desviación estándar; TUA=trastornos por uso de alcohol; TUS= trastornos por uso de sustancias.

Valor p es el nivel de significación calculado por: ( $\left.{ }^{1}\right)$ prueba T de Student y ( ${ }^{2}$ ) prueba exacta de Fisher ó chi-cuadrado.

$\left.{ }^{\star}\right)$ Se excluyen los trastornos por uso de sustancias (TUS)

número de criterios del episodio de máxima severidad. Estas diferencias también son muy significativas $(* \mathrm{p}<0,001)$ con respecto al género en la presencia de comorbilidad, donde los hombres también tienen más criterios [media 8,0 criterios $(\mathrm{DE}=2,3)$ ] que las mujeres, al comparar los dos grupos de comorbilidad psiquiátrica.
Añadir que se vieron diferencias de género en la presencia de síntomas físicos graves de dependencia, de convulsiones y/o delirium tremens, con una prevalencia del 7,4\% solo en hombres, sin que influya la presencia o no de otra comorbilidad psiquiátrica distinta al TUA. 
Nuria García-Marchena, Pedro Araos, Francisco Javier Pavón, Guillermo Ponce, María Pedraz, Antonia Serrano, Francisco Arias,

Pablo Romero-Sanchiz, Juan Suárez, Antoni Pastor, Rafael de la Torre, Marta Torrens, Gabriel Rubio, Fernando Rodríguez de Fonseca

Tabla 2. Patrones de uso de alcohol en una población de consumidores de alcohol en tratamiento en función del sexo y el diagnóstico de comorbilidad psiquiátrica a lo largo de la vida.

\begin{tabular}{|c|c|c|c|c|c|c|}
\hline \multirow[t]{2}{*}{ Patrones de uso de alcohol } & \multirow[t]{2}{*}{$\begin{array}{c}\text { Total } \\
N=162\end{array}$} & \multicolumn{2}{|c|}{$\begin{array}{l}\text { Hombres } \\
\mathrm{N}=122\end{array}$} & \multicolumn{2}{|c|}{$\begin{array}{l}\text { Mujeres } \\
\mathrm{N}=40\end{array}$} & \multirow[t]{2}{*}{ Valor $\mathrm{p}$} \\
\hline & & $\begin{array}{l}\text { Sin comorbilidad } \\
\text { psiquiátrica* }\end{array}$ & $\begin{array}{l}\text { Con comorbilidad } \\
\text { Psiquiátrica* }\end{array}$ & $\begin{array}{l}\text { Sin comorbilidad } \\
\text { psiquiátrica* }\end{array}$ & $\begin{array}{l}\text { Con comorbilidad } \\
\text { psiquiátrica* }\end{array}$ & \\
\hline $\begin{array}{l}\text { Edad primer consumo alcohol } \\
\text { [media (DE)] }\end{array}$ & $16,8(4,2)$ & $17,30(5,4)$ & $15,92(3,6)$ & $16,2(2,4)$ & $18,2(3,2) b b$ & $<0,01$ \\
\hline $\begin{array}{l}\text { Edad consumo } \\
\text { problemático de alcohol } \\
\text { [media }(D E)]\end{array}$ & $28,5(11,5)$ & $29,32(11,9)$ & $25,73(9,8)$ & $34,4(13,3)$ & $32,5(12,9) b$ & $<0,05$ \\
\hline $\begin{array}{l}\text { Duración de consumo } \\
\text { problemático (años) } \\
\text { [media (DE)] }\end{array}$ & $14,9(7,8)$ & $17,2(9,1)$ & $15,3(6,9)$ & $12,0(8,2)$ & $11,2(6,3) b$ & $<0,01$ \\
\hline $\begin{array}{l}\text { Periodos de } \\
\text { abstinencia anteriores ** } \\
\text { [media (DE)] }\end{array}$ & $1,2(1,1)$ & $1,0(0,9)$ & $1,3(1,2)$ & $1,4(1,3)$ & $0,9(1,0)$ & 0,345 \\
\hline $\begin{array}{l}\text { Duración de la última } \\
\text { abstinencia (meses) } \\
\text { [media (DE)] }\end{array}$ & $11,4(17,5)$ & $6,4(7,2)$ & $13,0(20,0)$ & $9,6(11,7)$ & $12,0(19,6)$ & 0,584 \\
\hline $\begin{array}{l}\text { Criterios de adicción en el } \\
\text { episodio de máxima severidad } \\
\text { [media (DE)] }\end{array}$ & $7,1(2,2)$ & $6,4(1,8)$ & $8,0(2,3)$ aaa & $6,1(1,1)$ & $6,2(1,9) \mathrm{bbb}$ & $<0,001$ \\
\hline
\end{tabular}

Nota. Abreviaturas: $\mathrm{N}=$ número de sujetos; $\mathrm{DE}=$ desviación estándar

Valor $\mathrm{p}$ es el nivel de significación calculado a partir del análisis de la varianza por rangos de Kruskal-Wallis

(aa) p<o,001 comparado con el grupo "Hombres sin comorbilidad";

(b, bb, bbb) p<0,05, p<0,01 y p<0,001 comparado con el grupo “Hombres con comorbilidad”. Calculados con la prueba post-test de Dunn

${ }^{\star}$ ) Se excluyen los trastornos por uso de sustancias (TUS)

$(\star \star)$ mínimo 6 meses de abstinencia

Tabla 3. Diagnósticos de trastornos por uso de alcohol y otras sustancias (DSM-IV-TR) en función del género y diagnóstico de comorbilidad psiquiátrica a a lo largo de la vida en una población de consumidores de alcohol en tratamiento.

\begin{tabular}{|c|c|c|c|c|c|c|c|}
\hline \multirow[t]{2}{*}{ Variable } & & \multirow[t]{2}{*}{$\begin{array}{l}\text { Total } \\
N=162\end{array}$} & \multicolumn{2}{|c|}{$\begin{array}{c}\text { Hombres } \\
\mathrm{N}=122\end{array}$} & \multicolumn{2}{|c|}{$\begin{array}{c}\text { Mujeres } \\
\mathrm{N}=40\end{array}$} & \multirow{2}{*}{$\begin{array}{c}\text { Valor } \mathbf{p} \\
\text { Género } \\
\text { Comorbilidad }\end{array}$} \\
\hline & & & $\begin{array}{l}\text { Sin comorbilidad } \\
\text { psiquiátrica* }\end{array}$ & $\begin{array}{c}\text { Con comorbilidad } \\
\text { psiquiátrica* }\end{array}$ & $\begin{array}{l}\text { Sin comorbilidad } \\
\text { psiquiátrica* }\end{array}$ & $\begin{array}{c}\text { Con comorbilidad } \\
\text { psiquiátrica* }\end{array}$ & \\
\hline $\begin{array}{l}\text { Alcohol } \\
{[N(\%)]} \\
\end{array}$ & $\begin{array}{l}\text { Abuso y/o } \\
\text { Dependencia }\end{array}$ & $162(100)$ & $44(36,1)$ & $78(63,9)$ & $7(17,5)$ & $33(82,5)$ & - \\
\hline $\begin{array}{l}\text { Cocaína } \\
{[N(\%)]}\end{array}$ & $\begin{array}{l}\text { Abuso y/o } \\
\text { Dependencia }\end{array}$ & $48(29,6)$ & $6(14,3)$ & $36(85,7)$ & - & $6(100)$ & $<0,001$ \\
\hline $\begin{array}{l}\text { Cannabis } \\
{[\mathrm{N}(\%)]}\end{array}$ & $\begin{array}{l}\text { Abuso y/o } \\
\text { Dependencia }\end{array}$ & $22(13,6)$ & $2(11,1)$ & $16(88,9)$ & - & $4(100)$ & $<0,05$ \\
\hline $\begin{array}{l}\text { Sedantes } \\
{[N(\%)]}\end{array}$ & $\begin{array}{l}\text { Abuso y/o } \\
\text { Dependencia }\end{array}$ & $6(3,7)$ & $1(25,0)$ & $3(75,0)$ & - & $2(100)$ & 0,543 \\
\hline $\begin{array}{l}\text { Otros } \\
\text { estimulantes** } \\
{[\mathrm{N}(\%)]}\end{array}$ & $\begin{array}{l}\text { Abuso y/o } \\
\text { Dependencia }\end{array}$ & $7(4,3)$ & $2(33,3)$ & $4(66,7)$ & - & $1(100)$ & 0,627 \\
\hline
\end{tabular}

Nota. Abreviaturas: $\mathrm{N}=$ número de sujetos; $\mathrm{DE}=$ desviación estándar.

Valor p es el nivel de significación calculado por prueba exacta de Fisher/chi-cuadrado agrupando los pacientes por género ó comorbilidad psiquiátrica.

Valor $\mathrm{p}$ es el nivel de significación calculado por prueba exacta de Fisher/chi-cuadrado agrupando los pacientes por género y comorbilidad.

$\left({ }^{\star}\right)$ Se excluyen los trastornos por uso de sustancias (TUS).

$\left({ }^{\star *}\right)$ Metanfetaminas y derivados.

\section{Comorbilidad psiquiátrica TUS. Abuso y dependencia de sustancias}

En la Tabla 3 se recoge la comorbilidad TUS de la población estudiada, en relación a la comorbilidad psiquiátrica y al género. Se pueden apreciar diferencias significativas $\left({ }^{*} \mathrm{p}<0,05\right)$ en los TUS de cocaína en relación con el géne- ro, siendo este trastorno más prevalente entre los hombres. Encontramos diferencias muy significativas $(* p<0,001)$ en la prevalencia de trastornos comórbidos en pacientes con TUS de cocaína, en comparación con los pacientes que no presentan TUS de cocaína. Se ven también diferencias significativas $(* \mathrm{p}<0,05)$ en relación al uso de cannabis, siendo ele- 
Tabla 4. Descripción de la comorbilidad psiquiátrica (DSM-IV-TR) a lo largo de la vida en una población de consumidores de alcohol en tratamiento.

\begin{tabular}{|c|c|c|c|c|c|}
\hline Variable & & $\begin{array}{r}\text { Total } \\
\mathrm{N}=162\end{array}$ & $\begin{array}{r}\text { Hombres } \\
\mathrm{N}=122\end{array}$ & $\begin{array}{r}\text { Mujeres } \\
\mathrm{N}=40\end{array}$ & Valor $p$ \\
\hline \multirow{7}{*}{$\begin{array}{l}\text { Comorbilidad } \\
\text { psiquiátrica* } \\
{[N(\%)]}\end{array}$} & Algún trastorno psiquiátrico & $111(68,5)$ & $78(63,9)$ & $33(82,5)$ & $<0,05$ \\
\hline & TEA & $74(45,7)$ & $47(38,5)$ & $27(67,5)$ & $<0,05$ \\
\hline & TA & $32(19,8)$ & $23(18,9)$ & $9(22,5)$ & 0,650 \\
\hline & Trastornos psicóticos & $15(9,3)$ & $10(8,2)$ & $5(12,5)$ & 0,529 \\
\hline & TCA & $2(1,2)$ & - & $2(5,0)$ & 0,060 \\
\hline & Trastornos de personalidad & $39(24,1)$ & $28(22,9)$ & $11(25,0)$ & 0,670 \\
\hline & TDAH & $46(28,4)$ & $37(30,3)$ & $9(22,5)$ & 0,421 \\
\hline $\begin{array}{l}\text { Trastornos del estado } \\
\text { de ánimo (TEA) } \\
\text { [N (\%)] }\end{array}$ & $\begin{array}{l}\text { Primaria } \\
\text { Inducida } \\
\text { Primaria+Inducida }\end{array}$ & $\begin{array}{r}30(18,5) \\
32(19,8) \\
12(7,4)\end{array}$ & $\begin{array}{r}19(15,6) \\
22(18,0) \\
6(4,9)\end{array}$ & $\begin{array}{r}11(27,5) \\
10(25,0) \\
6(15,0)\end{array}$ & 0,516 \\
\hline $\begin{array}{l}\text { Trastornos de ansiedad (TA) } \\
{[\mathrm{N}(\%)]}\end{array}$ & $\begin{array}{l}\text { Primaria } \\
\text { Inducida } \\
\text { Primaria+Inducida }\end{array}$ & $\begin{array}{r}19(11,7) \\
11(6,8) \\
2(1,2)\end{array}$ & $\begin{array}{r}12(9,8) \\
9(7,4) \\
2(1,6)\end{array}$ & $\begin{array}{r}7(17,5) \\
2(5,0) \\
-\end{array}$ & 0,422 \\
\hline $\begin{array}{l}\text { Trastornos psicóticos } \\
{[\mathrm{N}(\%)]}\end{array}$ & $\begin{array}{l}\text { Primaria } \\
\text { Inducida } \\
\text { Primaria+Inducida }\end{array}$ & $\begin{array}{l}5(3,1) \\
9(5,6) \\
1(0,6)\end{array}$ & $\begin{array}{l}4(3,3) \\
6(4,9) \\
-\end{array}$ & $\begin{array}{l}1(2,5) \\
3(7,5) \\
1(2,5)\end{array}$ & 1,000 \\
\hline $\begin{array}{l}\text { Trastornos de la conducta } \\
\text { alimentaria (TCA) } \\
\text { [N (\%)] }\end{array}$ & $\begin{array}{l}\text { Anorexia } \\
\text { Bulimia }\end{array}$ & $2(1,2)$ & - & $2(5,0)$ & 0,060 \\
\hline $\begin{array}{l}\text { Trastorno conducta infancia } \\
\text { [N (\%)] }\end{array}$ & & $14(8,6)$ & $13(10,7)$ & $1(2,5)$ & 0,192 \\
\hline $\begin{array}{l}\text { Trastornos de la personalidad } \\
{[\mathrm{N}(\%)]}\end{array}$ & $\begin{array}{l}\text { Trastorno antisocial } \\
\text { Trastorno límite }\end{array}$ & $\begin{array}{r}7(4,3) \\
24(14,8)\end{array}$ & $\begin{array}{r}7(5,7) \\
15(12,3)\end{array}$ & $9(22,5)$ & 0,152 \\
\hline \multicolumn{2}{|c|}{ Trastorno por déficit de atención e hiperactividad (TDAH) ${ }^{\star \star}[\mathrm{N}(\%)]$} & $24(14,8)$ & $19(15,6)$ & $5(12,5)$ & 0,799 \\
\hline
\end{tabular}

Nota. Abreviaturas: $\mathrm{N}=$ número de sujetos; $\mathrm{DE}=$ desviación estándar

Valor $p$ es el nivel de significación calculado por prueba exacta de Fisher/chi-cuadrado agrupando los pacientes por género

${ }^{\star}$ ) Eje I=Trastornos clínicos [se excluyen los trastornos por uso de sustancias (TUS)]; Eje II=Trastornos de personalidad

$\left({ }^{\star \star}\right)$ Diagnóstico TDAH (WURS)

vada la prevalencias de TUS en cannabis en otros trastornos de comorbilidad psiquiátrica, sin que existan diferencias debidas al género. Los pacientes con TUA y comorbilidad TUS de cannabis y cocaína, tienen más prevalencia de presentar comorbilidad a otros trastornos no relacionados con sustancias, que los pacientes sin comorbilidad TUS.

Debemos decir que para el resto de otras sustancias estudiadas no se encuentran diferencias estadísticamente significativas inter grupos a lo largo de la vida.

\section{Comorbilidad psiquiátrica de trastornos no relaciona- dos con uso de sustancias}

En la Tabla 4 se recogen los distintos diagnósticos excluyendo los trastornos relacionados con uso de sustancias que han presentado los pacientes, recogidos con los instrumentos de evaluación (PRISM y WURS) y diferen- ciados por género. Encontramos diferencias estadísticamente significativas $(* \mathrm{p}<0,05)$ entre la prevalencia de trastornos psiquiátricos, con un $82,5 \%$ de mujeres frente a un $63,9 \%$ de hombres con un diagnóstico de trastorno mental distinto del adictivo. Se encuentran también diferencias muy significativas $(* \mathrm{p}<0,01)$ en la prevalencia de TEA en mujeres $(67,5 \%)$ y hombres $(38,5 \%)$. En los demás trastornos no encontramos diferencias significativas debidas al género.

\section{Diferencias de género en comorbilidad psiquiátrica y por TUS}

En este apartado (ver Tabla 5), queremos ver en detalle la distribución de trastornos psiquiátricos entre las diferencias existentes debidas al género y el tipo de TUS diagnosticado. Podemos decir que, cuando diferenciamos según las sustan- 
Nuria García-Marchena, Pedro Araos, Francisco Javier Pavón, Guillermo Ponce, María Pedraz, Antonia Serrano, Francisco Arias,

Pablo Romero-Sanchiz, Juan Suárez, Antoni Pastor, Rafael de la Torre, Marta Torrens, Gabriel Rubio, Fernando Rodríguez de Fonseca

Tabla 5. Descripción de la comorbilidad psiquiátrica (DSM-IV-TR) a lo largo de la vida en una población de consumidores de alcohol en tratamiento agrupada por género y diagnóstico de trastornos por uso de alcohol y de otras sustancias.

\begin{tabular}{|c|c|c|c|c|c|c|}
\hline \multirow[t]{2}{*}{ Variables } & \multirow[t]{2}{*}{ Total } & \multicolumn{2}{|l|}{$\begin{array}{l}\text { Hombres } \\
\mathrm{N}=122\end{array}$} & \multicolumn{2}{|l|}{$\begin{array}{l}\text { Mujeres } \\
\mathrm{N}=40\end{array}$} & \multirow[t]{2}{*}{ Valor $p$} \\
\hline & & TUA + otra TUS & TUA & TUA + otra TUS & TUA & \\
\hline $\begin{array}{l}\text { Número de pacientes } \\
{[\mathrm{N}(\%)]}\end{array}$ & $162(100)$ & $53(43,4)$ & $69(56,6)$ & $10(25,0)$ & $30(75,0)$ & $<0,05$ \\
\hline $\begin{array}{l}\text { Comorbilidad psiquiátrica * } \\
{[\mathrm{N}(\%)]}\end{array}$ & $111(68,5)$ & $46(37,7)$ & $32(26,2)$ & $10(25,0)$ & $23(57,5)$ & $<0,01$ \\
\hline $\begin{array}{l}\text { Comorbilidad psiquiátrica } \\
\text { ( } \geq \text { dos diagnósticos) * } \\
{[\mathrm{N}(\%)]}\end{array}$ & $63(38,9)$ & $31(25,4)$ & $14(11,5)$ & $8(20,0)$ & $10(25,0)$ & 0,087 \\
\hline $\begin{array}{l}\text { Trastornos del } \\
\text { estado de ánimo (TEA) } \\
\text { [N (\%)] }\end{array}$ & $74(45,7)$ & $24(19,7)$ & $23(18,9)$ & $8(20,0)$ & $19(47,5)$ & $<0,001$ \\
\hline $\begin{array}{l}\text { Trastornos psicóticos } \\
\text { [N (\%)] }\end{array}$ & $15(9,3)$ & $5(4,1)$ & $5(4,1)$ & $2(5,0)$ & $3(7,5)$ & 1,000 \\
\hline $\begin{array}{l}\text { Trastornos de la } \\
\text { conducta alimentaria (TCA) } \\
\text { [N (\%)] }\end{array}$ & $2(1,2)$ & - & - & $2(5,0)$ & - & - \\
\hline $\begin{array}{l}\text { Trastorno de la } \\
\text { personalidad antisocial } \\
\text { [N (\%)] }\end{array}$ & $11(6,8)$ & $9(7,4)$ & $1(0,8)$ & $1(2,5)$ & - & - \\
\hline $\begin{array}{l}\text { Trastorno de la } \\
\text { personalidad límite } \\
\text { [N (\%)] }\end{array}$ & $28(17,3)$ & $13(10,6)$ & $5(4,1)$ & $7(17,5)$ & $3(7,5)$ & 1,000 \\
\hline $\begin{array}{l}\text { Trastorno por déficit } \\
\text { de atención } \\
\text { e hiperactividad (TDAH) ** } \\
{[\mathrm{N}(\%)]}\end{array}$ & $46(28,4)$ & $28(22,9)$ & $9(7,4)$ & $4(10)$ & $5(12,5)$ & 0,106 \\
\hline
\end{tabular}

Nota. Abreviaturas: $\mathrm{N}=$ número de sujetos; TUA=trastornos por uso de alcohol; TUS=trastornos por uso de otras sustancias

Valor p es el nivel de significación calculado por prueba exacta de Fisher/chi-cuadrado agrupando los pacientes por género y por diagnóstico de TUA y TUS

(*) Eje I=Trastornos clínicos [se excluyen los trastornos por uso de sustancias (TUS)]; Eje II=Trastornos de personalidad.

$(\star \star)$ Diagnóstico TDAH (WURS)

cias, la comorbilidad psiquiátrica varía entre los que tienen solamente un trastorno de uso de alcohol (TUA), frente a los que además del TUA presentan otro TUS. En el caso de las mujeres con comorbilidad TUS, vemos como el $100 \%$ presentan además otros trastornos psiquiátricos a lo largo de la vida, siendo más prevalentes los TEA. Los hombres sin comorbilidad TUS (solo TUA), presentan menor prevalencia de otros trastornos psiquiátricos si los comparamos con aquellos que tienen comorbilidad TUS, y aunque estas diferencias no se aprecian en el diagnóstico de TEA, son muy marcadas en los trastornos de personalidad.

Hacemos una revisión especial al trastorno por déficit de atención e hiperactividad, por su estrecha relación con las sustancias depresoras y su alta prevalencia en TUS (Daigre et al., 2013; Polanczyk et al., 2014), en TUA, al igual que sucede en nuestros pacientes (Ponce et al., 2000).

\section{Valores plasmáticos de 2-acilgliceroles}

Los niveles plasmáticos de 2-acilgliceroles se ven reducidos en pacientes con comorbilidad psiquiátrica (excluyendo los TUS) sin que existan diferencias significativas entre los grupos respecto a la comorbilidad psiquiátrica. Hemos distinguido temporalidad entre los pacientes con comorbilidad psiquiátrica (a lo largo de la vida y en el último año) y comparado con pacientes sin comorbilidad psiquiátrica (Figura 1).

Los niveles plasmáticos de 2-AG y 2-OG están disminuidos significativamente $\left({ }^{*} \mathrm{p}<0,05\right)$ en pacientes con diagnóstico de trastornos de ansiedad en el último año cuando comparamos sus concentraciones con pacientes con trastornos de ansiedad a lo largo de la vida, sin diagnósticos de trastornos de ansiedad y con pacientes sin comorbilidad psiquiátrica (Figura 2). 

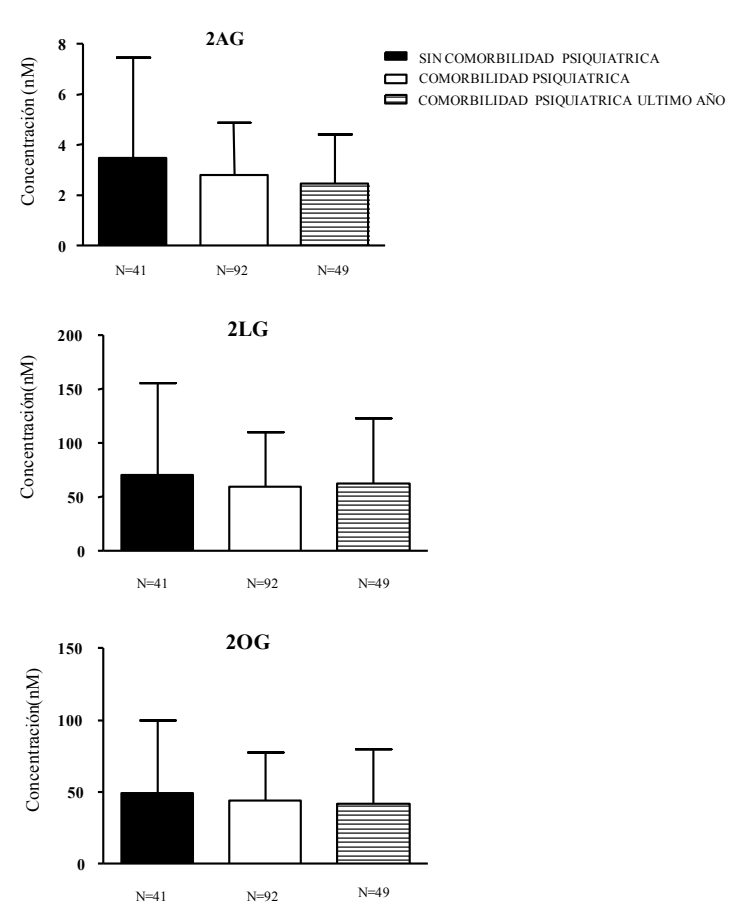

(\#) Se excluyen los trastornos por uso de sustancias (TUS)

Figura 1. Niveles plasmáticos de 2-acilgliceroles [2-araquidonilglicerol (2-AG), 2-linoleilglicerol (2-LG) y 2-oleoilglicerol (2-OG)] en consumidores de alcohol en tratamiento agrupados por comorbilidad psiquiátrica (\#)
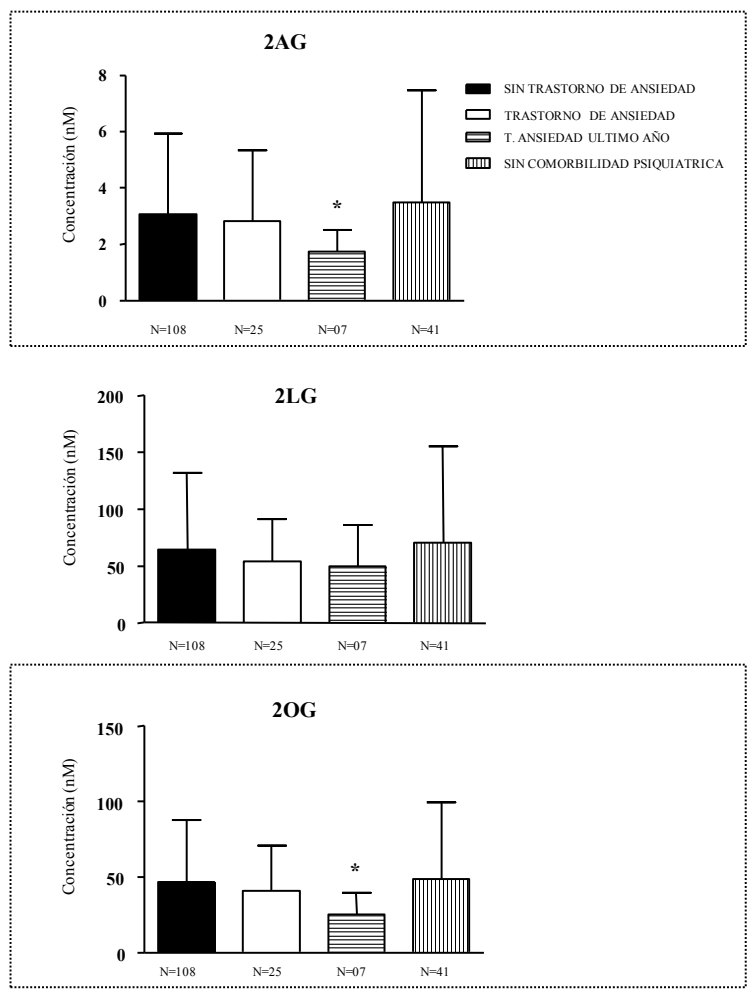

$\left(^{\star}\right)$ pro.05 indica diferencias significativas respecto al grupo sin trastorno de ansiedad

Figura 2. Niveles plasmáticos de 2-acilgliceroles [2-araquidonilglicerol (2-AG), 2-linoleilglicerol (2-LG) y 2-oleoilglicerol (2-OG)] en consumidores de alcohol en tratamiento agrupados por diagnósticos de trastornos de ansiedad (DSM-IV-TR).
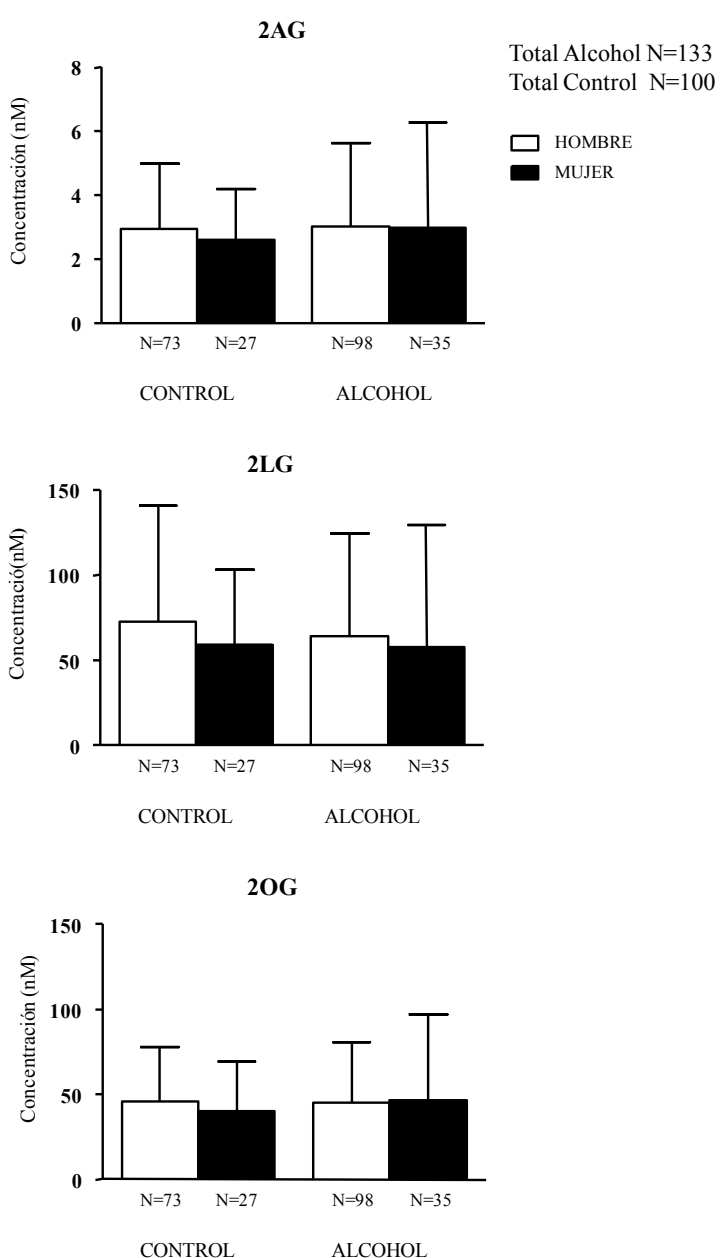

Figura 3. Niveles plasmáticos de 2-acilgliceroles [2-araquidonilglicerol (2-AG), 2-linoleilglicerol (2-LG) y 2-oleoilglicerol (2-OG)] en consumidores de alcohol en tratamiento agrupado por sexo y comparado con población control.

Los niveles de 2-acilgliceroles no muestran diferencias significativas respecto al sexo cuando comparamos la muestra de pacientes con TUA con un grupo control (Figura 3).

\section{Discusión}

Los resultados del presente estudio indican que los pacientes que solicitan tratamiento presentan una mayor comorbilidad psiquiátrica y que en algunos casos, en especial en relación con los trastornos de ansiedad, esta se refleja en niveles alterados de 2-acilgliceroles. La prevalencia de comorbilidad psiquiátrica de este estudio a lo largo de la vida ha sido alta $(68,5 \%)$ en un rango similar a algunos estudios realizados sobre distintos tipos de muestra con TUA y otras patologías, con 60-70\% de comorbilidad psiquiátrica (Pérez-Gálvez et al., 2008; Driessen et al., 1998). Nuestro per- 
fil de paciente es principalmente masculino, con un nivel educativo medio-bajo, de edad media 49,27 años y un alto grado de desempleo. Población en la que se excluyen los casos de diagnóstico con trastorno mental grave pero no las patologías orgánicas. Estas características pueden suponer una serie de sesgos que hay que tener en cuenta a la hora de comparar nuestros datos con otras poblaciones con TUA.

Existen diferencias importantes de género dentro del TUA. Sería interesante destacar que aunque las mujeres comienzan a beber a edades más avanzadas que los hombres, tardan menos tiempo que ellos en desarrollar criterios de dependencia al alcohol y cumplen menos criterios de severidad que los hombres. Estos datos se asemejan a los encontrados en la bibliografía, donde las mujeres tienen un patrón de consumo intenso, con un progreso más rápido desde el inicio del consumo hasta la dependencia. Este patrón podría ser la causa de que tengan mayor prevalencia de problemas médicos concomitantes en comparación con los hombres con TUA (Ávila Escribano et al., 2007; Keyes et al., 2010). También se aprecian diferencias de género en los trastornos no relacionados con sustancias, donde ellos tienen mayores prevalencias. El trastorno antisocial de la personalidad es diagnosticado tres veces más en hombres que en mujeres (Alegría et al., 2013). Las mujeres tienen mayores prevalencias en los trastornos depresivos (Ávila Escribano et al., 2007). La falta de apoyo social en las mujeres y los problemas interpersonales en el contexto familiar, serían factores de riesgo y posible causa de las diferencias de género en la comorbilidad psiquiátrica (Alegría et al., 2013).

En el análisis de la población con respecto a la comorbilidad debida a un TUS, encontramos que el policonsumo podría estar relacionado con la comorbilidad psiquiátrica, especialmente cuando el TUA va acompañado de otros TUS de sustancias como cocaína y cannabis. Sabemos que los TUS por cocaína están asociados a una elevada prevalencia de comorbilidad psiquiátrica (Araos et al., 2014) y además encontramos en la literatura una elevada prevalencia de policonsumo en pacientes en seguimiento que son mayoritariamente hombres jóvenes, nunca casados, con una edad temprana de inicio del consumo y comorbilidad psiquiátrica (Blanco et al., 2015). Si analizamos la comorbilidad psiquiátrica en nuestra muestra encontramos que, primero, los trastornos de estado de ánimo primarios y los inducidos tuvieron similar prevalencia a lo largo de la vida. Sabemos que la depresión que comienza antes del inicio del TUS reduce las posibilidades de remisión de la dependencia, al igual que lo hacen las depresiones mayores inducidas (Samet et al., 2013). Algunos estudios aseguran que el grado de severidad del TUS es un buen predictor de trastornos depresivos en el seguimiento del paciente (Boschloo et al., 2012), esta afirmación tiene sentido en nuestra muestra al ver relación entre presencia de comorbilidad psiquiátrica y severidad de criterios de adicción en TUA. Segundo, respecto a los trastornos de ansiedad, en nuestro estudio fueron más prevalentes los trastornos primarios que los inducidos para los diagnósticos a lo largo de la vida. Tercero, los trastornos psicóticos en nuestra muestra, son mayoritariamente inducidos aunque distan mucho de los encontrados en otras poblaciones, en la que las prevalencias fueron elevadas $(15,5 \%)$ (Araos et al., 2014). Aunque los trastornos del estado de ánimo y de ansiedad primarios son mayoritariamente más frecuentes que los inducidos por sustancias (Torrens et al., 2011), nuestros resultados concuerdan con otros estudios realizados mediante la evaluación PRISM en poblaciones con TUS por cocaína, donde los trastornos inducidos fueron más prevalentes que los primarios (Araos et al., 2014; Vergara-Moragues et al., 2012). Por último, sobre los TP, existe una prevalencia del trastorno antisocial de la personalidad de $6,8 \%$ y un $17,4 \%$ de trastorno límite de la personalidad en nuestra población, prevalencias más bajas que las encontradas en poblaciones con TUS de otras sustancias como cocaína (Araos et al., 2014). Hay evidencias que sugieren que los trastornos de personalidad se asocian más a otros TUS, por ser el consumo de estas las que aumentan los problemas conductuales, la severidad clínica y las dificultades sociales (Salom et al., 2014).

El diagnóstico de TDAH tienen una prevalencia del 7\% en la infancia y de ellos aproximadamente el $4 \%$ prevalece en la edad adulta (Kessler et al., 2006). Nuestra población tiene una elevada prevalencia en este trastorno, en estos resultados puede influir la utilización de un instrumento específico para su diagnóstico. Aun así, los síntomas en el adulto pueden dar lugar a errores, como que la inquietud se interprete como ansiedad o la distraibilidad por falta de interés o motivación (Quintero et al., 2013). El hecho de tener comorbilidad TUS aumenta la comorbilidad psiquiátrica de la muestra (Tómasson y Vaglum, 1995). En nuestra muestra, cuando eliminamos la presencia de otros TUS en los pacientes con TUA, la prevalencia de otra comorbilidad psiquiátrica se reduce (de $82,5 \%$ a $57,5 \%$ en mujeres y de $63,9 \%$ a $26,2 \%$ en hombres). Disminuye también la prevalencia de trastornos de personalidad, porque el trastorno antisocial en hombres sin comorbilidad TUS baja a $0,8 \%$ y el trastorno límite en mujeres al 7,5\%. Obtenemos cifras que concuerdan con estudios descritos en poblaciones similares en nuestro país, en el que el trastorno antisocial tendría prevalencias del $2 \%$ y el trastorno límite de la personalidad $6 \%$ (Fernández-Montalvo et al., 2006). Estos pacientes experimentan dificultades de comportamiento tanto internos como externos en comparación con lo que solo cursan TUA (Salom et al., 2014).

Los resultados sobre comorbilidad psiquiátrica arriba descritos apoyan la necesidad de buscar pruebas biológicas objetivas que sirvieran como biomarcadores específicos de subgrupos con comorbilidades psiquiátricas específicas. A este respecto, nuestro estudio aporta la descripción por primera vez de los valores plasmáticos 2-acilgliceroles en estos pacientes. Los datos sugieren que el 2-AG y 2-OG están 
afectados por diagnósticos de trastornos de ansiedad en el último año. La señalización endocannabinoides se ha implicado en la modulación de la ansiedad y la respuesta emocional (Navarro et al., 1997). Un incremento de la liberación de endocannabinoides se asocia a efecto ansiolítico, lo que ha motivado el desarrollo de múltiples fármacos, especialmente aquellos relacionados con el bloqueo de la actividad de las enzimas degradadoras de endocannabinoides FAAH y MAGL (Gaetani et al., 2003; Kinsey et al., 2011). Encontramos estudios que relacionan la disregulación de acilgliceroles con la dificultad de adaptación a estímulos estresantes y aversivos con un aumento de respuestas de estrés, ansiedad y miedo (Guggenhuber et al., 2015; Jenniches et al., 2015). El hecho de que nosotros encontremos también una relación entre cambios en 2- acilgliceroles y los diagnósticos de trastornos de ansiedad, nos sitúa más cerca de posibles dianas terapéuticas de estrés y trastornos relacionados con la ansiedad.

Entre nuestras limitaciones hay que destacar que la evaluación retrospectiva de la sintomatología clínica y de abstinencia es imperfecta, así como los periodos de esta última relatados por los pacientes. Por otro lado, el tamaño de la muestra, relativamente pequeño desde una perspectiva estadística, aunque relevante desde un punto de vista clínico, así como el porcentaje de población femenina estudiado.

Las perspectivas futuras a considerar pasan por continuar con este tipo de estudios de caracterización fenotípica con una muestra más amplia y representativa. Desarrollar un trabajo longitudinal que permita comprobar la evolución de los síntomas psicopatológicos y de severidad en la adicción y ampliar la búsqueda de población femenina que cumpla criterios de inclusión con la finalidad de hacer comparaciones de género más profundas y diseñar tratamientos de atención especializada, la necesidad de incorporar y aumentar la muestra de distintos biomarcadores de diagnóstico en consumo, severidad y comorbilidad en la realización de mejoras en pronóstico y tratamientos óptimos adaptados a las necesidades de cada perfil de paciente.

\section{Reconocimientos}

A cada paciente que participó en el estudio, por su tiempo y esfuerzo. Agradecer también al servicio de psiquiatría del 12 de Octubre de Madrid su colaboración, especial dedicación y profesionalidad a todos ellos, médicos, enfermeras, técnico de laboratorio y administrativos.

El presente estudio ha sido financiado por Instituto de Salud Carlos III (ISC-III), Red de Trastornos Adictivos UE-FEDER 2012 (RD12/0028); Ministerio de Economía y Competitividad (PI13/02261); Plan Nacional sobre Drogas 049/2009 y 049/2013; Consejería de Economía, Innovación y Ciencia, Junta de Andalucía UE-FEDER (CTS-433); Consejería de Salud y Bienestar Social, Junta Andalucía
(PI0228-2013 y PI0823-2012). Contratos "Miguel Servet" del Instituto de Salud Carlos III y Fondo Europeo de Desarrollo Regional FEDER/EU-ERDF (CP14/00212 y CP14/00173). Contrato "Río Hortega" del Instituto de Salud Carlos III (CM13/0115).

\section{Conflicto de intereses}

No existen conflictos de intereses.

\section{Referencias}

Alegría, A. A., Blanco, C., Petry, N. M., Skodol, A. E., Liu, S. M., Grant, B. y Hasin, D. (2013). Sex differences in antisocial personality disorder: results from the national epidemiological survey on alcohol and related conditions. Personal Disorders, 4, 214-222. doi: 10.1037/a0031681.

Araos, P., Vergara-Moragues, E., Pedraz, M., Pavón, F.J., Campos, R., Calado, M.,... Rodríguez de Fonseca, F. (2014). Comorbilidad psicopatológica en consumidores de cocaína en tratamiento ambulatorio. Adicciones, 26, 15-26.

Ávila Escribano, J. J. y González Parra, D. (2007). Gender differences in alcoholism. Adicciones, 19, 383-392.

Basavarajappa, B. S. y Hungrund, B. L. (2005). Role of the endocannabinoid system in the development of tolerance to alcohol. Alcohol and Alcoholism, 40, 15-24.

Blanco, C., Iza, M., Rodríguez-Fernández, J. M., Baca-García, E., Wang, S. y Olfson, M. (2015). Probability and predictors of treatment-seeking for substance use disorders in the U.S. Drug and Alcohol Dependence, 149, 136144. doi: 10.1016/j.drugalcdep.2015.01.031.

Caillé, S., Alvarez-Jaimes, L. Polis, I., Stouffer, D. G. y Parsons, L. H. (2007). Specific alterations of extracellular endocannabinoid levels in the nucleus accumbens by ethanol, heroin, and cocaine self-administration. The Journal of Neuroscience, 27, 3695-3702.

Boschloo, L., Van den Brink, W., Penninx, B. W. J. H., Wall, M. M. y Hasin, D. S. (2012). Alcohol-use disorder severity predicts first-incidence of depressive disorders. Psychological Medicine, 42, 695-703. doi: 10.1017/ S0033291711001681.

Driessen, M., Veltrup, C., Wetterling, T., John, U. y Dilling, H. (1998). Axis I and Axis II comorbidity in alcohol dependence and the two types or alcoholism). Alcoholism: Clinical and Experimental Research, 22, 77-86.

Daigre, C., Terán, A., García-Vicent, V. y Roncero, C. (2013). Trastorno por déficit de atención con hiperactividad y dependencia de sustancias depresoras. Adicciones, 25, 171-186.

Engelhard, C. P., Touquet, G., Tansens, A. y De Fruyt, J. (2015). [Alcohol-induced psychotic disorder: a systematic literature review]. Tijdschrift voor Psychiatrie, 57, 192-201.

Fernández-Montalvo, J., Landa, N., López-Goñi, J. J. y Lorea, I. (2006). Personality disorders in alcoholics: A 
comparative pilot study between the IPDE and the MCMI-II. Addictive Behaviors, 31, 1442-1448.

Fiedler, K. K., Kim, N., Kondo, D. G. y Renshaw, P. F. (2012). Cocaine use in the past year is associated with altitude of residence. Journal of Addiction Medicine, 6, 166-171. doi: 10.1097/ADM.0b013e31824b6c62.

Flensborg-Madsen, T., Knop, J. Mortensen, E. L., Becker, U., Sher, L. y Gronbaek, M. (2009). Alcohol use disorders increase the risk of completed suicide-irrespective of other psychiatric disorders. A longitudinal cohort study. Psychiatry Research, 167, 123-130. doi: 10.1016/j. psychres.2008.01.008.

Gaetani, S., Cuomo, V. y Piomelli, D. (2003). Anandamide hydrolysis: a new target for anti-anxiety drugs?. Trends in Molecular Medicine, 9, 478-478.

Grant, B. F., Stinson, F. S., Dawson, D. A., Chou, S. P., Ruan, W. J. y Pickering, R. P. (2004). Co-occurrence of 12-Month Alcohol and Drug Use Disorders and PersonalityDisorders in the United States: Results From the National Epidemiologic Survey on Alcohol and Related Conditions. Archives of General Psychiatry, 61, 361-368.

Goldsmith, R. J. (1999). Overview of psychiatric comorbidity: practical and theoretic considerations. Psychiatric Clinics of North America, 22, 331-349.

Guggenhuber, S., Romo-Parra, H., Bindila, L., Leschik, J., Lomazzo, E., Remmers, F.,... Lutz, B. (2015). Impaired 2-AG signaling in hippocampal glutamatergic neurons: aggravation of anxiety-Like behavior and unaltered seizure susceptibility. International Journal of Neuropsychopharmacology, pyv091. doi: 10.1093/ijnp/pyv091.

Hasin, D. S., Trautman, K. D., Miele, G. M., Samet, S., Smith, M. y Endicott, J. (1996). Psychiatric Research Interview for Substance and Mental Disorders (PRISM): Reliability for substance abusers. American Journal of Psychiatry, 153, 1195-1201.

Hasin, D. S., Goodwin, R. D., Stinson, F. S. y Grant, B. F. (2005). Epidemiology of Major Depressive Disorder: Results from the National Epidemiologic Survey on Alcoholism and Related Conditions. Archives of General Psychiatry, 62, 1097-1106.

Hasin, D. S., Stinson, F. S., Ogburn, E. y Grant, B. F. (2007). Prevalence, correlates, disability, and comorbidity of DSM-IV alcohol abuse and dependence in the United States: results from the National Epidemiologic Survey on Alcohol and Related Conditions. Archives of General Psychiatry, 64, 830-842.

Jenniches, I., Ternes, S., Albayram, O., Otte, D. M., Bach, K., Bindila, L.,... Zimmer, A. (2015). Anxiety, stress and fear response in mice with reduced endocannabinoid levels. Biological Psychiatry. doi: 10.1016/j. biopsych.2015.03.033.

Karila, L., Petit, A., Lowenstein, W. y Reynaud, M. (2012). Diagnosis and consequences of cocaine addiction. $\mathrm{Cu}$ rrent Medical Chemistry, 19, 5612-5618.
Karila, L., Zarmdini, R., Petit, A., Lafaye, G., Lowenstein, W. y Reynaud, M. (2014). Cocaine addiction: Current data for clinician. Presse Medicale 43, 9-17.

Kessler, R. C., Crum, R. M., Warner, L. A., Nelson, C. B., Schulenberg, J. y Anthony, J. C. (1997). Lifetime co-occurrence of DSM-III-R alcohol abuse and dependence with other psychiatric disorders in the National Comorbidity Survey. Archives of General Psychiatry, 54, 313-321.

Kessler, R. C., Adler, L., Barkley, R., Biederman, J., Conners, C. K., Delmer, O.,... Zaslavsky, A. M. (2006). The Prevalence and Correlates of Adult ADHD in the United States: Results From the National Comorbidity Survey Replication. American Journal of Psychiatry, 163, 716723.

Kessler, R. C. y Ustün, T. B. (2004). The word mental health $(\mathrm{WMH})$ survey initiative version of the world health organization (WHO) composite international diagnostic interview (CIDI). International Journal of Methods in Psychiatric Research, 13, 93-121.

Keyes, K. M., Martins, S. S., Blanco, C. y Hasin D. S. (2010). Telescoping and gender differences in alcohol dependence: new evidence from two national surveys. American Journal Psychiatry, 167, 969-976. doi: 10.1176/appi. ajp.2009.09081161.

Kinsey, S. G., O’Neal, S. T., Long, J. Z., Cravatt, B. F. y Lichtman, A. H. (2011). Inhibition of endocannabinoid catabolic enzymes elicits anxiolytic-like in the marble burying assay. Pharmacology Biochemistry and Behavior, 98, 21-27. doi: 10.1016/j.pbb.2010.12.002.

Malinen, H., Lehtonen, M. y Hyytiä, P. (2009). Modulation of brain endocannabinoid levels by voluntary alcohol consumption in alcohol-preferring AA rats. Alcoholism: Clinical and Experimental Research, 33, 1711-1720. doi: 10.1111/j.1530-0277.2009.01008.x.

Morguello, S., Holzer, C.E., Ryan, E., Young, C., Naseer, M., Castellon, S. A.,... Singer, E. J. (2006). Interrater reliability of the Psychiatric Research Interview for Substance and mental Disorders in an HIV-infected cohort: experience of the National NeuroAIDS Tissue Consortium. International Journal of Methods in Psychiatric Research, 15, 131-138.

Navarro, M., Hernández, E., Muñoz, R. M., del Arco, I., Villanúa, M. A., Carrera, M. R. y Rodríguez de Fonseca, F. (1997). Acute administration of the CB1 cannabinoid receptor antagonist SR 141716A induces anxiety-like responses in the rat. Neuroreport, 8, 491-496.

Observatorio Español sobre Drogas (2013). Encuesta Domiciliaria sobre Alcohol y Drogas en España (EDADES) 2011-2012.

Pavón, F. J., Araos, P., Pastor, A., Calado, M., Pedraz, M., Campos-Cloute, R.,... Rodríguez de Fonseca, F. (2013). Evaluation of plasma-free endocannabinoids and their congeners in abstinent cocaine addicts seeking outpatient treatment: impact of psychiatric co-morbidity. $A d-$ diction Biology, 18, 955-969. doi: 10.1111/adb.12107. 
Pedraz, M., Araos, P., García-Marchena, N., Serrano, A., Romero-Sanchiz, P, Suárez, J.,... Pavón, F.J. (2015). Sex differences in psychiatric comorbidity and plasma biomakers for cocaine addiction in abstinent cocaine-addicted subjects in outpatient settings. Frontiers in Psychiatry, 16, 6-17. doi: 10.3389/fpsyt.2015.00017.

Pérez-Gálvez, B., de Vicente-Manzanaro, M. P., García-Fernández, L., Romero-Escobar, C. y Oliveras-Valenzuela, M. A. (2008). Prevalencia de psicopatología en dependientes al alcohol en tratamiento ambulatorio. Trastornos Adictivos, 10, 42-48.

Piomelli, D. (2003). The molecular logic of endocannabinoid signalling. Nature Reviews Neuroscience, 4, 873-884.

Polanczyk, G. V., Willcut, E. G., Salum, G. A., Kieling, C. y Rohde, L. A. (2014). ADHD prevalence estimates across three decades: an updated systematic review and meta-regression analysis. International Journal of Epidemiology, 43, 434-442. doi: 10.1093/ije/dyt261.

Ponce, G., Rodríguez-Jiménez, R., Rojo, J.A., Sánchez-Monasor, R. y Rubio, G. (2000). Attention-defict hyperactivity disorder and vulnerability to the development of alcoholism: use of the Wender-Utah Rating Scale for retrospective diagnostic of ADHD in the childhood of alcoholic patients. Actas Españolas de Psiquiatría, 28, 357-366.

Quintero, J., Balanzá-Martínez, V., Correas, J. y Soler, B. (2013). Attention Deficit Hyperactivity Disorder (ADHD) in the adult patients: view of the clinician. Actas Españolas de Psiquiatría, 41, 185-195.

Robins, L. N., Wing, J., Wittchen, H. U., Helzer, J. E., Babor, T. F., Burke, J.,... Towle, L. H. (1988). The Composite International Diagnostic Interview: an epidemiologic instrument suitable for use in conjunction with different diagnostic systems and in different cultures. Archives of General Psychiatry, 45, 1069-1077.

Rodríguez-Jiménez, R., Ponce, G., Monasor, R., Jiménez-Giménez, M., Pérez-Rojo, J.A. y Rubio, G. (2001). Validación en población española adulta de la Wender-Utah Rating Scale para la evaluación retrospectiva de trastorno por déficit de atención e hiperactividad en la infancia. Revista de Neurología, 33, 44.

Rodriguez-Jimenez, R., Aragües, M., Jiménez-Arriero, M. A., Ponce, G., Muñoz, A., Bagney, A.,... Palomo, T. (2008). Dual diagnosis in psychiatric inpatients: prevalence and general characteristics. Investigacion Clínica, 49, 195-205.

Ross, H. E. (1995). DSM-III-R alcohol abuse and dependence and psychiatric comorbidity in Ontario: results from the Mental Health Supplement to the Ontario Health Survey. Drug and Alcohol Dependence, 39, 111128.

Ruffles, G. (2009). Emergency care for young people who have used cocaine. Emergency Nurse, 17, 34-36. doi: http:/ /dx.doi.org/10.7748/en2009.12.17.8.34.c7433
Salom, C. L., Betts, K. S., Williams, G. N., Najman, J. N., Scott, J. G. y Alati, R. (2014). Do young people with comorbid mental and alcohol disorders experience worse behavioral problems?. Psychiatry Research. 219, 372-379. doi: 10.1016/j.psychres.2014.05.056.

Samet, S., Fenton, M., Nunes, E., Greenstein, E., Aharonovich, E. y Hasin, D. (2013). Effects of independent and substance-induced major depressive disorder on remission and relapse of alcohol, cocaine and heroin dependence. Addiction, 108, 115-123. doi: 10.1111/j.13600443.2012.04010.x.

Serrano, A., Rivera, P., Pavón, F. J., Decara, J., Suárez, J., Rodríguez de Fonseca F. y Parsons L. H. (2012). Differential effects of single versus repeated alcohol withdrawal on the expression of endocannabinoid system-related genes in the rat amygdala. Alcoholism: Clinical and Experimental Research 36, 984-994. doi: 10.1111/j.15300277.2011.01686.x.

Tómasson, K. y Vaglum, P. (1995). A nationwide representative sample of treatment seeking alcoholics: A study of psychiatric comorbidity. Acta Psychiatrica Scandinavica, 92, 378-385.

Torrens, M., Serrano, D., Astals, M., Pérez-Domíngez, G. y Martín-Santos, R. (2004). Diagnosis comorbid psychiatric disorders in substance abusers: validity of the Spanish versions of the Psychiatric Research Interview for Substance and Mental Disorders and the Structured Clinical Interview for DSM-IV. American Journal Psychiatry, 161, 1231-1237.

Torrens, M., Gilchrist, G., y Domingo-Salvany, A. y psyCoBarcelona Group (2011). Psychiatric comorbidity in illicit drug users: Substance-induced versus independent disorders. Drug and Alcohol Dependence. 133, 147-156. doi: 10.1016/j.drugalcdep.2010.07.013.

Vergara-Moragues, E., González-Saiz, F., Lozano, O. M., Betanzos Espinosa, P., Fernández Calderón, F., Bilbao-Acebos, I.,... Verdejo García, A. (2012). Psychiatric comorbidity in cocaine users treated in therapeutic community. Substance induced versus independent disorders. Psychiatry Research, 200, 734-741. doi: 10.1016/j. psychres.2012.07.043.

World Health Organization (2014). Global status report on alcohol and health-2014. World Health Organization. 